\title{
Detection of Sinkhole Attacks for Mobile Nodes in Heterogeneous Sensor Networks with Mobile Sinks
}

\author{
P. Samundiswary,Padma Priyadarshini and P. Dananjayan
}

\begin{abstract}
Sensor networks consist of large number of nodes with limited energy, computation and transmission power. Since these nodes operate in a physically insecure environment, they are vulnerable to different types of attacks such as selective forwarding and sinkhole. These attacks can inject malicious packets by compromising the nodes. Furthermore, previous work on sensor networks mainly considered homogeneous sensor networks where all sensor nodes have the same capabilities. Generally homogeneous sensor networks have poor performance limits and scalability. Hence, the network performance can be improved by deploying heterogeneous sensor networks. However, security mechanism is not explored for mobile nodes with sink mobility in heterogeneous sensor network with limited memory against the above mentioned attacks. In this paper, a secured path redundancy algorithm is attempted to implement in heterogeneous sensor networks by incorporating alternate path scheme in heterogeneous sensor networks with mobile nodes for mobile sinks to defend against sinkhole attacks. The simulation results prove that this algorithm has better network performance in terms of energy consumption, delivery ratio and delay than that of heterogeneous sensor networks in the presence of compromised nodes.
\end{abstract}

Keywords-Wireless Sensor Networks; Homogeneous Sensor Networks; Heterogeneous Sensor Networks; Malicious Nodes; Selective forwarding Attacks; Sinkhole Attacks; Mobile Sinks.

\section{INTRODUCTION}

Wireless sensor networks are expected to have applications in many areas, such as military, homeland security, environmental monitoring and healthcare systems. Most research work in sensor networks considers homogeneous sensor networks, where all sensor nodes are modeled to have the same capabilities in terms of computation, communication, energy supply, storage, reliability and other aspects [1]. Security is critical for sensor networks deployed in hostile environments to defend against attacks. Providing security and privacy to small sensor nodes is challenging, due to the limited capabilities of sensor nodes. However, only few of the routing protocols such as security protocols for sensor networks (SPINS) and path redundancy based security

P. Samundiswary is with Department of Electronics and Communication Engineering,Sri Manakula Vinayagar Engineering

College,Pondicherry-605107, India(email: samundiswary_pdy@yahoo.com)

Padma Priyadarshini and P. Dananjayan are with Department of Electronics and Communication Engineering,Pondicherry Engineering College,Pondicherry-605014, India(pdananjayan@ rediffmail.com) algorithm (PRSA) for wireless sensor networks address the security mechanism and authentication against the various attacks [2,3]. Moreover, secured homogeneous sensor networks suffer from poor fundamental limit and performance. To achieve better performance, a heterogeneous sensor network (HSN) model is adopted. The HSN consists of a few powerful high-end sensors (H-sensors) and a large number of low-end sensors (L-sensors). Compared to the $\mathrm{L}$-sensor, the $\mathrm{H}$-sensor has better computation capability, larger storage and better reliability [4]. The sensor nodes may be captured by attackers and reloaded with malicious nodes. Some of the secured routing protocols of heterogeneous sensor networks can detect the malicious nodes and deliver the packets to the sink successfully. But these routing protocols increase the buffering requirements and delay [5]. In this paper, PRSA is extended for heterogeneous sensor networks by including alternate path mechanism and mobility model for nodes and sinks to protect the nodes from sinkhole attacks in HSN. This algorithm is simulated by using Glomosim for different coverage area of $300 \mathrm{~m} \times 300 \mathrm{~m}$ and $500 \mathrm{~m} \times 500 \mathrm{~m}$ considering mobile nodes and mobile sinks in heterogeneous sensor networks against sinkhole attacks.

The remainder of the paper is organized as follows Section 2 describes about the heterogeneous sensor network model. Section 3 deals with the proposed secured path redundancy algorithm for heterogeneous sensor network. Simulation results are discussed in Section 4 to obtain energy consumption, delay and delivery ratio of the proposed security scheme and conclusion are drawn in Section 5.

\section{Heterogeneous SENSOR NETwORKS}

In the HSN model, H-sensors and L-sensors are randomly distributed in the field and clusters are formed. The cluster formation is shown in Fig.1, where L-sensors are the small squares, H-sensors are large rectangles, and the large square at the top-right corner is the base station (BS). It is natural to let powerful $\mathrm{H}$-sensors serve as cluster heads The $\mathrm{H}$-sensors have more energy supply, longer transmission range, higher data rate than L-sensors. All the $\mathrm{H}$-sensors form a backbone in the network. H-sensors use multi-hop communications to reach the base station (BS) and L-sensors also use multi-hop communications to reach $\mathrm{H}$-sensors [6].H-sensors provide many advantages for designing better protocols, algorithms, and secure schemes in sensor networks. An efficient and 
robust cluster formation scheme with well organized routing protocol is adopted for HSNs.

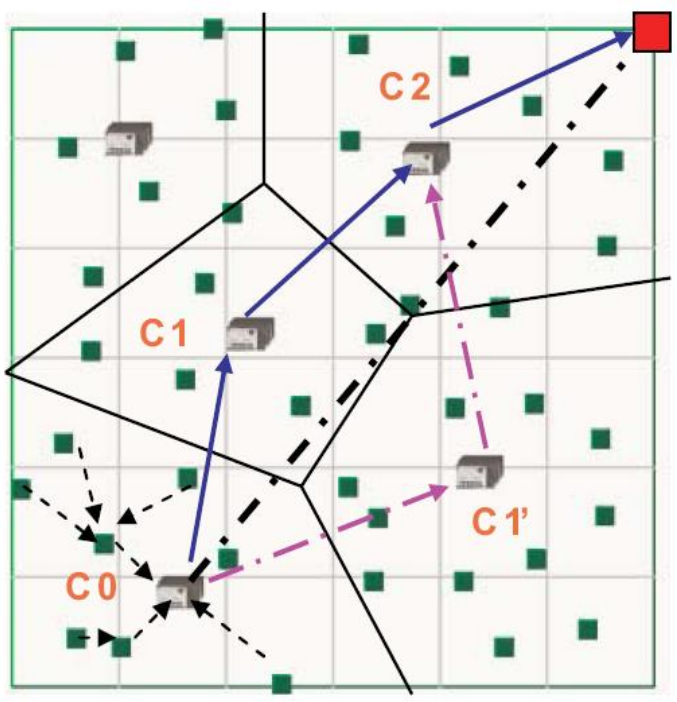

Heterogeneous sensor network model

\section{A. Routing in Heterogeneous Sensor Networks}

The primary functionality of a wireless sensor network is to sense the environment and transmit the acquired information to the BS for further processing. Since sensor nodes are small and unreliable devices, they are prone to failures Thus, routing is an essential operation in sensor networks. A routing protocol should be robust to sensor failures and be able to find new paths when nodes fail. Security requirement adds new challenges to routing. Recent literatures have shown that routing protocols of HSNs can significantly improve the performance of sensor networks. The basic idea of routing in HSNs is to let each L-sensor sends data to its cluster head (an H-sensor). An H-sensor may aggregate data from multiple L-sensors and removes redundant data, and then send compressed data to the $\mathrm{BS}$ via the H-sensor backbone. Transmissions in the backbone have longer range and may use a different frequency than transmissions among L-sensors. Based on the above two layer communication architecture, an efficient routing protocol is implemented for HSNs. This consists of two parts: routing within a cluster (among L-sensors), and routing across clusters (among H-sensors) [7]. Each type of routing of HSN is discussed below.

\section{B. Intra-Cluster Routing}

Routing within a cluster (from an L-sensor to its cluster head) is referred to as intra-cluster routing [7] which is illustrated in Fig.1. L-sensor sends its location information to the cluster head (H-sensor) during the cluster formation. The location of $\mathrm{H}$ is broadcasted to all L-sensors in the cluster. All the L-sensors in a cluster form a tree, rooted at the cluster head (denoted as $\mathrm{H}$ ) so that each L-sensor sends packets to its $\mathrm{H}$-sensor, when it generates packets. If data from nearby L-sensor nodes are highly correlated, then a minimum spanning tree (MST) can be adopted to approximate the least energy consumption case. A centralized algorithm created by $\mathrm{H}$-sensor can be used to construct an MST. Then $\mathrm{H}$ disseminates the MST structure information to L-sensors, i.e., informing each L-sensor which node its parent is. If a data fusion is conducted at intermediate L-sensors nodes (two $\mathrm{k}$-bit packets come in, and one $k$-bit packet goes out after data fusion), then MST consumes the least total energy in the cluster. If there is few or no data fusion among L-sensors in a cluster, a shortest-path tree (SPT) should be used to approximate the least total energy consumption. Similarly, the cluster head (H-sensor) can construct an SPT by using a centralized algorithm and the locations of L-sensors. Since L-sensors are small, unreliable devices, they may fail over time. Therefore robust and self-healing routing protocols are critical for routing among L-sensors. In the above route setup, each L-sensor may record two or more parent nodes. One parent node serves as the primary parent, and other parent nodes serve as backup parents. If the primary parent node fails, an L-sensor can use a backup parent for data forwarding. Further each L-sensor records one or more backup cluster heads during cluster formation. When a cluster head fails, L-sensors in the cluster send their packets to a backup cluster head.

\section{Inter-Cluster Routing}

Routing across clusters (from an $\mathrm{H}$-sensor to the BS) is referred to as inter-cluster routing which is shown in Fig.1. After receiving data from L-sensors, cluster heads may perform data aggregation via the $\mathrm{H}$-sensor backbone. Each cluster head exchanges location information with neighbor cluster heads. During route discovery, a cluster head draws a straight line $L$ between itself and the BS, based on the locations of the BS and itself which is shown in Fig.1. Line $L$ intersects with a serial of clusters, and these clusters are denoted as $C_{0}, C_{1}, \ldots, C_{k}$, which are referred to as Relay Cells. The packet is forwarded from the source cluster head to the BS via cluster heads in the Relay Cells. H-sensors are more reliable nodes than L-sensors. However, an H-sensor may also fail because of various reasons, such as harsh environment, or destroyed by an adversary. If any cluster head in the Relay Cells is unavailable, then a backup path is used. A backup path is set up as follows: The current cluster head (say $R 1$ ) draws a straight line $L$ ' between itself and the $\mathrm{BS}$, and line $L$ intersects with several cells $\mathrm{C}_{1}^{\prime}, \ldots, C^{\prime}{ }_{k-1}, C^{\prime}{ }_{k}$. If the next cell is the cell having the failed cluster head, $R 1$ will use a detoured path to avoid the cell. The sequence cells $C^{\prime}{ }_{1}, \ldots, C^{\prime}{ }_{k-1}, C^{\prime}{ }_{k}$ will be the new Relay Cell and are used to forward the packet to the BS.

\section{Secured Path Redundancy Algorithm For Mobile Nodes With Sink Mobility In Heterogeneous SENSOR NETWORKS}

This algorithm may be applied for a network with various numbers of nodes; network capacity and attack type. The nodes are assumed to be mobile nodes in this network. The idea behind alternate path redundancy algorithm is to find secure multiple paths between the source and destination nodes in the presence of sinkhole attackers. adversary goal of the sinkhole attacks is to lure nearly all the traffic from a particular area through a compromised node, creating a metaphorical sinkhole with the adversary at the centre[8]. A 
sinkhole attack is a type of attack which has more than one malicious node of attackers makes a compromised node looks more attractive to surrounding nodes by forging routing information [9]. The end result is that surrounding nodes will choose the compromised node as the next node to route the data through. This is achieved by removing one or more nodes that is suspected to be an active adversary node from the routing path. Such nodes are identified by algorithm using a set of parameters that is usually reflecting the presence of adversary nodes [3]. The parameters used are packet ID, no. of hop counts and delay to reach the destination. This secured mechanism can defend against attacks such as sinkhole attacks.

Further more, sink mobility brings new challenges to data dissemination in large sensor networks. Sink mobility suggests that information about each mobile sink's location be continuously propagated throughout the sensor field in order to keep all sensor nodes is informed about the direction of forwarding future data reports. Unfortunately, frequent location updates from multiple sinks can lead to both excessive drain of sensors' limited battery supply and increased collisions in wireless transmissions [10]. To avoid these limitations, the same secured path redundancy algorithm for HSN approach is extended for mobile sinks which consumes less energy, better delay and efficient data delivery to more than one mobile sinks.

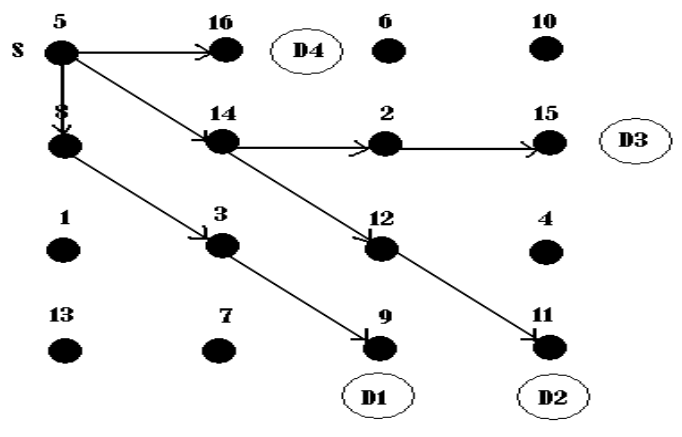

Mobile sink

\section{Simulation Results}

The secured path redundancy algorithm for mobile nodes with sink mobility in heterogeneous sensor network is simulated for two different coverage areas $(300 \mathrm{~m} \times 300 \mathrm{~m}$, $500 \mathrm{~m} \times 500 \mathrm{~m}$ ) by varying the number of nodes from 25 to 500 nodes with 10 to 50 numbers of malicious nodes in Glomosim. The number of mobile sinks ranges from 1 to 6 . The mobility model used for nodes and sink mobility in the simulation is random way point. The energy consumption, delivery ratio and delay are calculated for various numbers of nodes with two different coverage areas such as $300 \mathrm{~m} \times 300 \mathrm{~m}$, $500 \mathrm{~m} \times 500 \mathrm{~m}$ having different values of malicious nodes $(10$ to 50). The parameters used in this simulation are given in Table.1.

TABLE. 1 SIMULATION PARAMETERS

\begin{tabular}{|l|l|}
\hline Simulation parameters & Values \\
\hline Number of nodes & 25 to 500 \\
\hline Geographical area $\left(\mathrm{m}^{2}\right)$ & $300 \times 300,500 \times 500$ \\
\hline Packet Size(bytes) & 64 \\
\hline
\end{tabular}

\begin{tabular}{|l|l|}
\hline Traffic Type & CBR \\
\hline Number of malicious nodes & 10 to 50 \\
\hline Number of mobile sinks & 1 to 6 \\
\hline Mobility model & Random way point \\
\hline Simulation time(s) & 600 \\
\hline
\end{tabular}

\section{A. Energy Consumption}

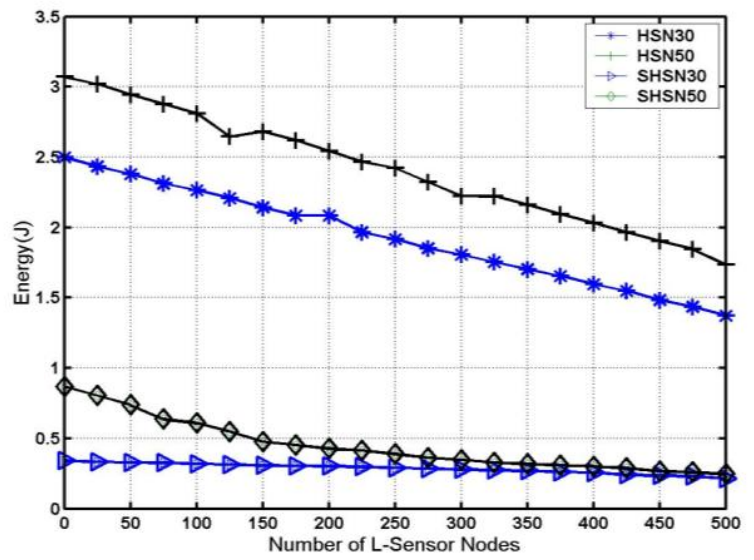

(a).Energy consumption Vs number of L-sensor nodes for coverage area $300 \mathrm{~m} \times 300 \mathrm{~m}$

The simulation results shown in Fig.3 (a) and Fig.3 (b) prove that there is a significant reduction in the energy consumption of secured heterogeneous sensor networks by increasing the numbers of nodes for different coverage area and different values of malicious nodes. Fig.3(c) shows that there is increment in the energy consumption of secured heterogeneous sensor networks for mobile nodes compared to that of secured heterogeneous sensor networks having fixed nodes in the presence of attackers. When the number of nodes increases, the energy consumption of secured heterogeneous sensor networks reduces from $57 \%$ to $81.5 \%$ compared to heterogeneous sensor networks of malicious node 30 with the coverage area of $300 \mathrm{~m} \times 300 \mathrm{~m}$. Fig.3 (d) and Fig .3(e) illustrate that when the number of mobile sinks increases, energy consumption of heterogeneous sensor networks reduces for different coverage areas. Even if the number of malicious nodes and coverage area increases, the energy consumption of secured network reduces by $49 \%$ to $67 \%$ with respect to heterogeneous sensor networks. Energy consumption of secured heterogeneous sensor networks is lesser than heterogeneous sensor networks for fixed and mobile nodes because nodes involve alternate shortest path and less number of broken paths by using $\mathrm{H}$-sensors even in the presence of malicious nodes. 


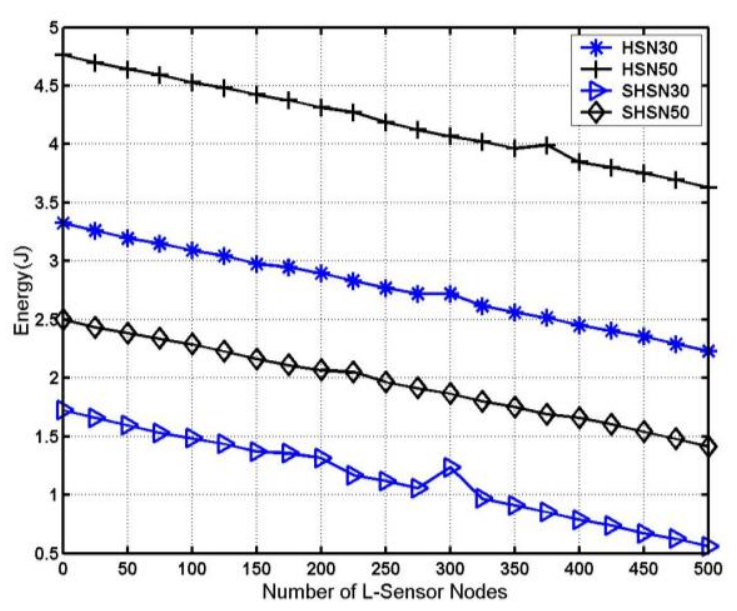

(b).Energy consumption Vs number of L- sensor nodes for coverage area $500 \mathrm{~m} \times 500 \mathrm{~m}$

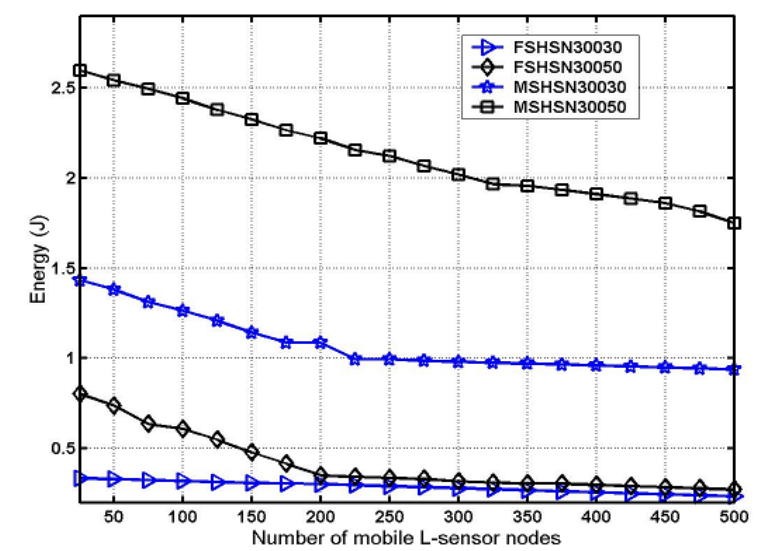

(c).Energy consumption Vs number of mobile L- sensor nodes for coverage area $300 \mathrm{~m} \times 300 \mathrm{~m}$

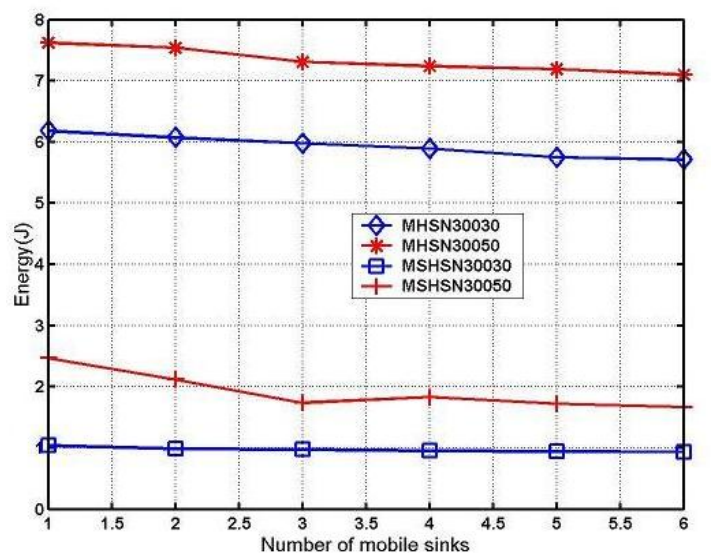

(d).Energy consumption Vs number of mobile sinks for coverage area $300 \mathrm{~m} \times 300 \mathrm{~m}$

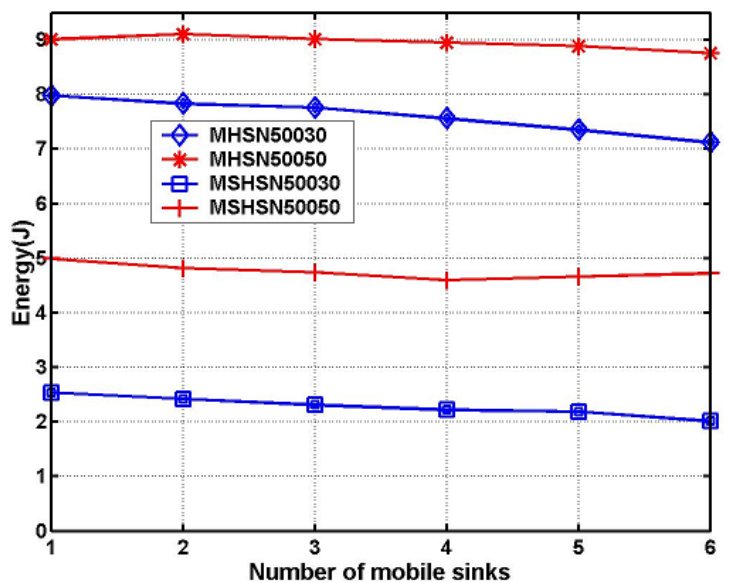

(e).Energy consumption Vs number of mobile sinks for coverage area $500 \mathrm{~m} \times 500 \mathrm{~m}$

\section{B. Delay}

The delay graph is illustrated in Fig.4 (a) and Fig.4 (b) considering fixed L-sensor nodes for different coverage areas with 30 and 50 malicious nodes. The graphs prove that secured path redundancy algorithm for heterogeneous sensor network of fixed nodes is lower than that of heterogeneous sensor network by $50 \%$ to $55 \%$ in case of 30 malicious nodes for network coverage area of $300 \mathrm{~m} \times 300 \mathrm{~m}$. The secured path redundancy algorithm for heterogeneous sensor networks of mobile nodes is also lesser than the secured heterogeneous sensor networks of fixed nodes which is shown in Fig.4(c) and Fig.4 (d). Fig.4 (e) shows that there is reduction in delay of secured heterogeneous network than that of heterogeneous sensor networks considering mobile nodes and sink mobility for different network area of $300 \mathrm{~m} \times 300 \mathrm{~m}$ and $500 \mathrm{~m} \times 500 \mathrm{~m}$. Since packets of secured path redundancy algorithm for heterogeneous sensor network use a secured path which require less hop count and delay to reach the mobile sinks from the source even in the presence of malicious nodes.

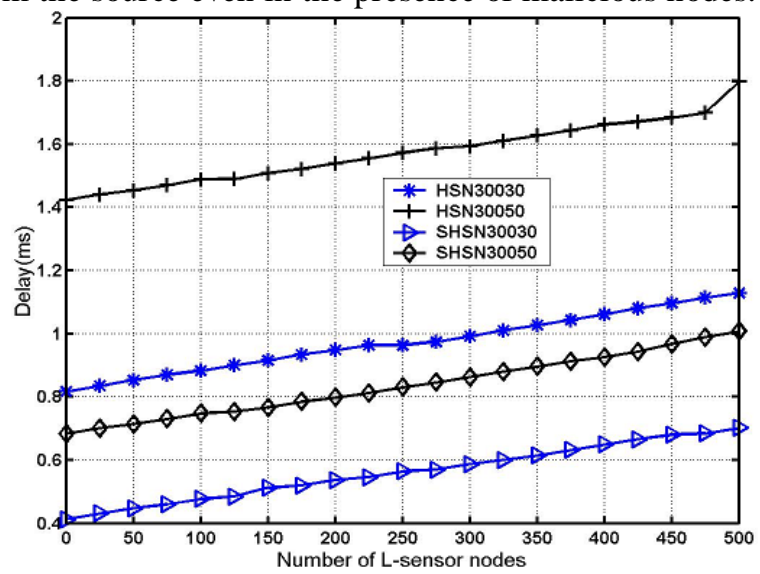

(a).Delay with respect to number of L-sensor nodes for coverage area $300 \mathrm{~m} \times 300 \mathrm{~m}$

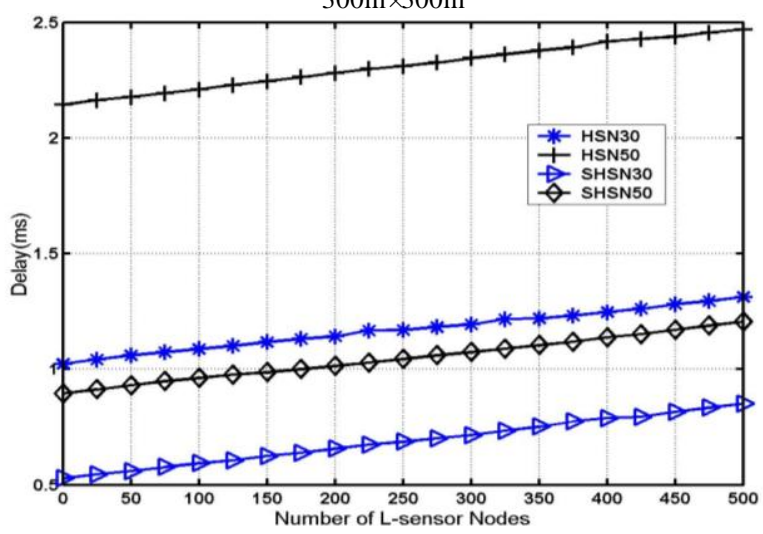

(b).Delay with respect to number of L-sensor nodes for coverage area $500 \mathrm{~m} \times 500 \mathrm{~m}$ 


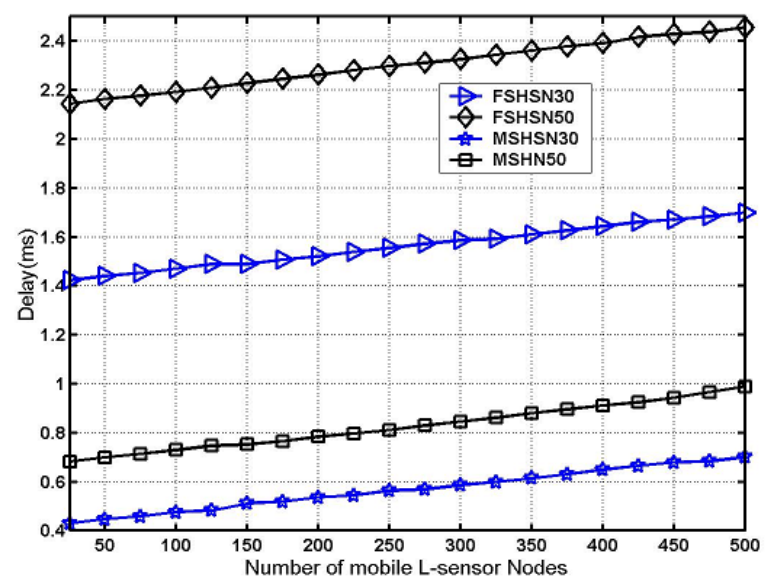

(c).Delay with respect to number of mobile $\mathrm{L}$ sensor nodes for coverage area $300 \mathrm{~m} \times 300 \mathrm{~m}$

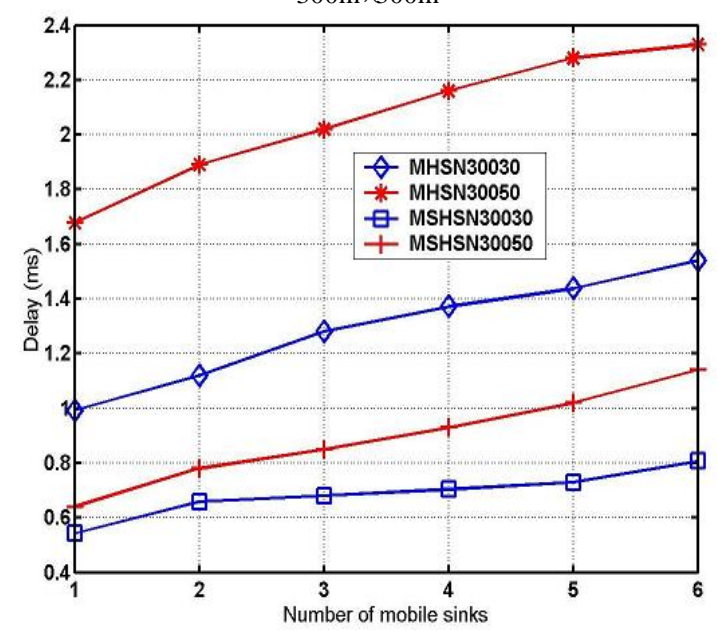

(d).Delay with respect to number of mobile sinks for coverage area $300 \mathrm{~m} \times 300 \mathrm{~m}$

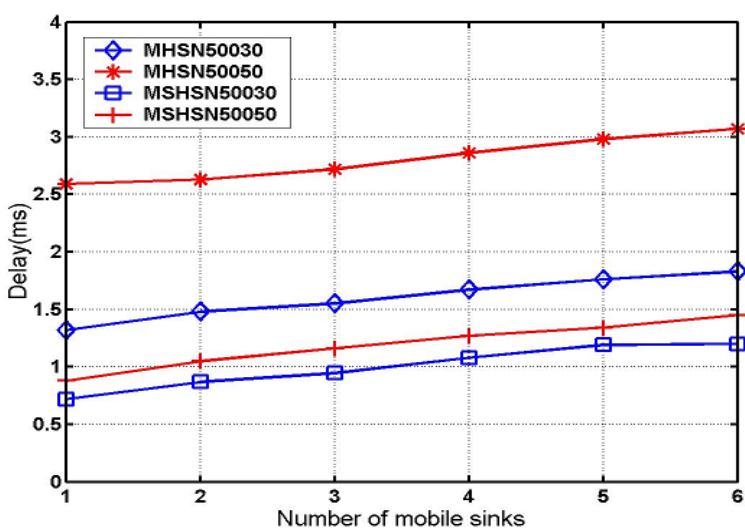

(e).Delay with respect to number of mobile sinks for coverage area $500 \mathrm{~m} \times 500 \mathrm{~m}$

\section{Delivery Ratio}

Delivery ratio of secured path redundancy algorithm for heterogeneous sensor networks is higher than heterogeneous sensor networks which is shown in Fig.5 (a) and Fig.5 (b). for different values of malicious nodes and different coverage area. In Fig.5(c), the delivery ratio of secured path redundancy algorithm of heterogeneous sensor networks of mobile nodes is higher than that of heterogeneous sensor network for fixed nodes in the presence of malicious nodes by $6 \%-10 \%$. Fig.5(d) illustrates that secured HSN delivers more numbers of packets from the source to more than one mobile sinks successfully compared to that of heterogeneous sensor networks even in the existence of compromised nodes. The fact is that secured HSNs packets require less number of hops from the L-sensors to the cluster head than HSN. Moreover, the packet loss is reduced due to secured path from source to sink in secured heterogeneous sensor network.

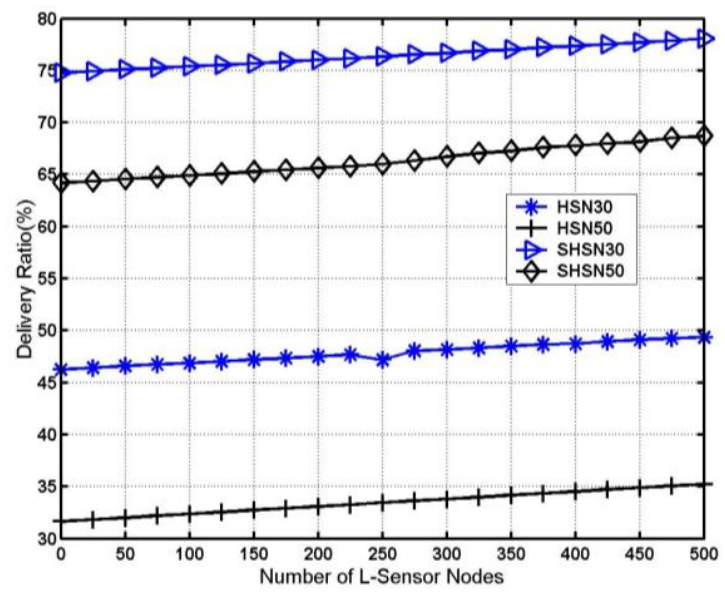

(a).Delivery ratio with respect to number of L-sensor nodes for coverage area $300 \mathrm{~m} \times 300 \mathrm{~m}$

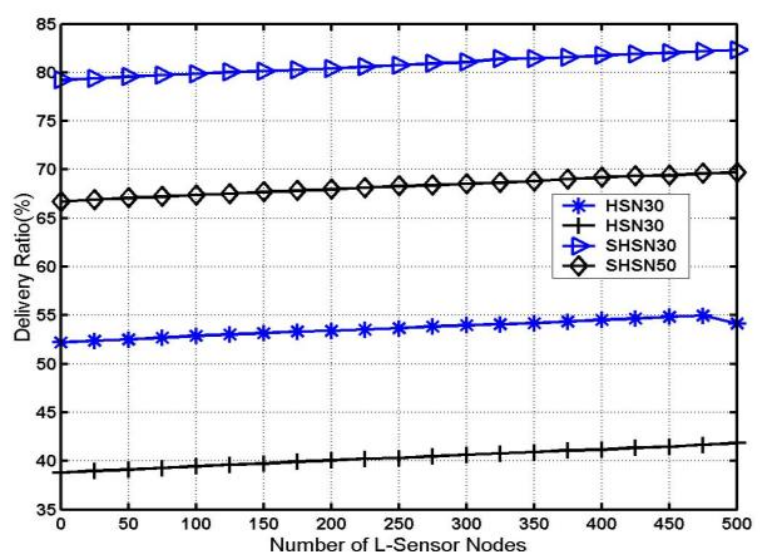

(b).Delivery ratio with respect to number of L-sensor nodes for coverage area $500 \mathrm{~m} \times 500 \mathrm{~m}$

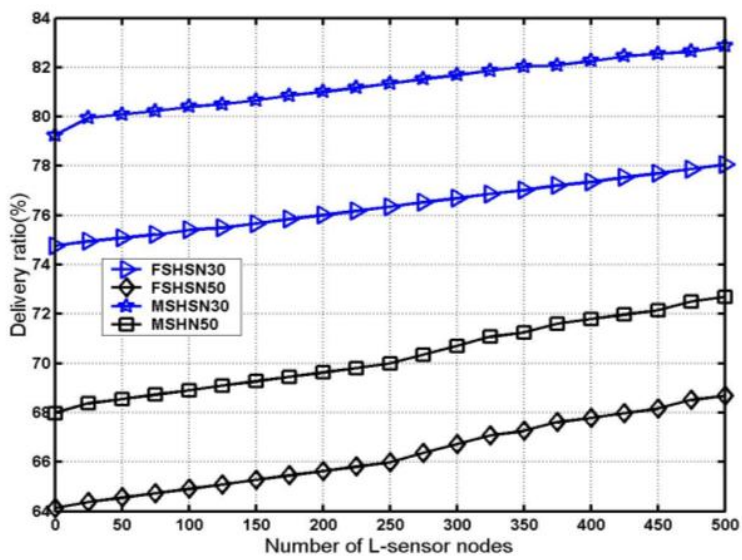

(c).Delivery ratio with respect to number of mobile sensor nodes for coverage area $300 \mathrm{~m} \times 300 \mathrm{~m}$ 


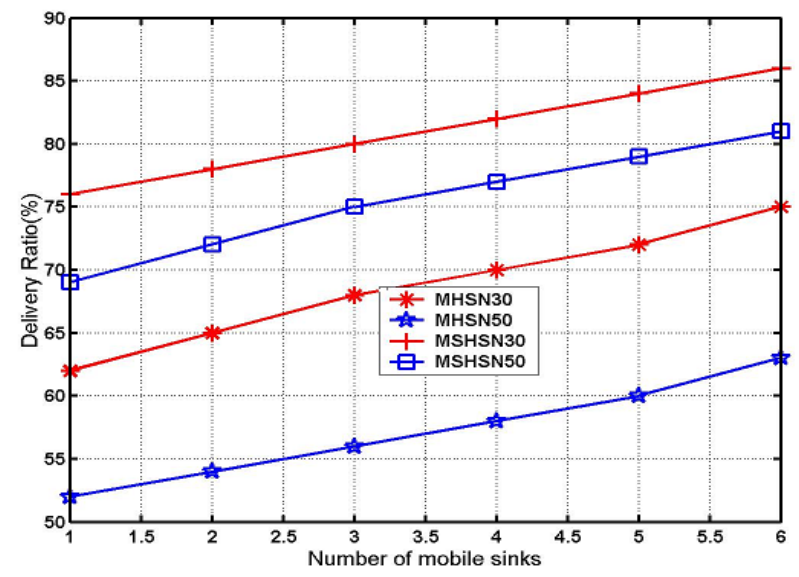

(d).Delivery ratio with respect to number of mobile sinks for coverage area $300 \mathrm{~m} \times 300 \mathrm{~m}$.

\section{Defending against sinkhole attacks}

The performance parameters such as energy and delay are improved by implementing this path redundancy algorithm in hetrogeneous sensor networks to secure these networks from sinkhole attacks for different coverage areas $(300 \mathrm{~m} \times 300 \mathrm{~m}$ and 500m $\times 500 \mathrm{~m})$. Fig.6(a) and Fig.6(c) depict the energy of various number of nodes for different values of malicious nodes with different coverage area scenarios. Eventhough there is an increment in the value of energy for higher number of malicious nodes, but there is reduction in energy when the number of mobile nodes increase by using secured path redundancy algorithm for heterogeneous sensor networks to shield against sinkhole attacks. Fig.6(b) and Fig.6(d) show the comparison of delay with respect to the number of mobile nodes for various number of malicious nodes considering coverage area of $300 \mathrm{~m} \times 300 \mathrm{~m}$ and $500 \mathrm{~m} \times$ $500 \mathrm{~m}$. Though the delay increases with respect to the number of nodes shown in Fig.6 (b) and Fig.6 (d), this algorithm for heterogeneous sensor networks is used to transmit the packets to the sink successfully in the presence of attackers.

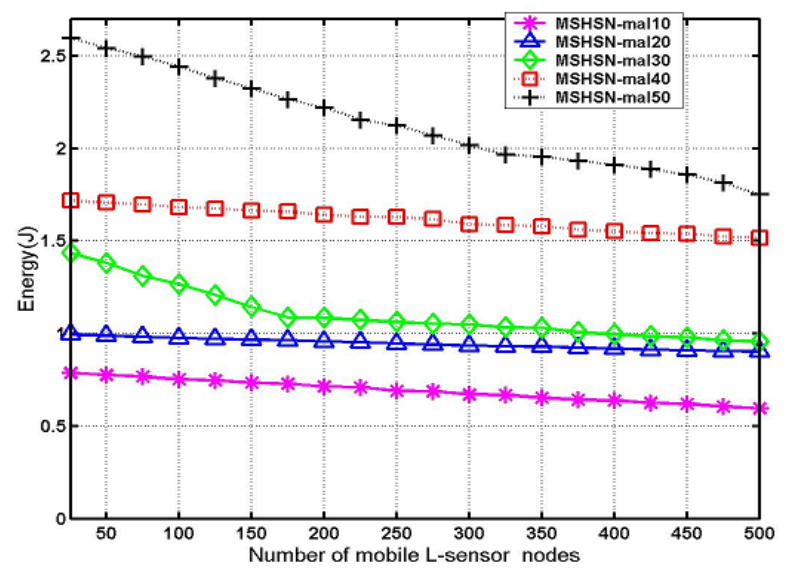

(a).Comparison of Energy Vs number of mobile nodes for various malicious nodes with coverage area $300 \mathrm{~m} \times 300 \mathrm{~m}$

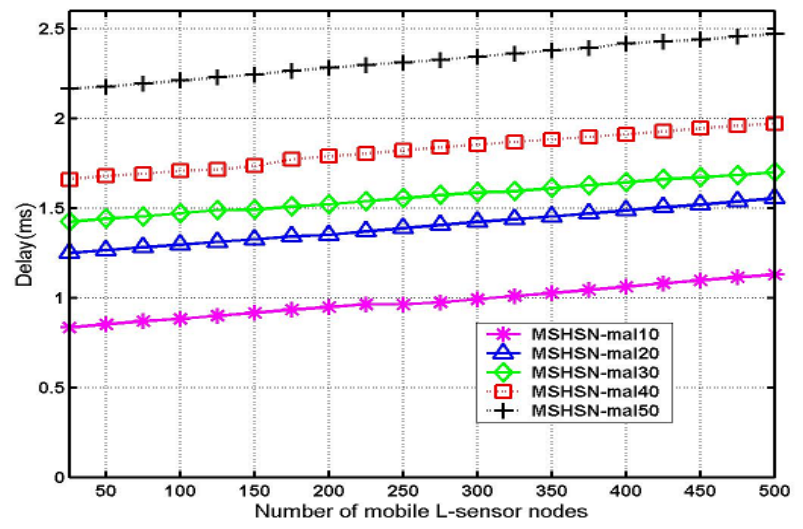

(b).Comparison of Delay Vs number of mobile nodes for various malicious nodes with coverage area $300 \mathrm{~m} \times 300 \mathrm{~m}$

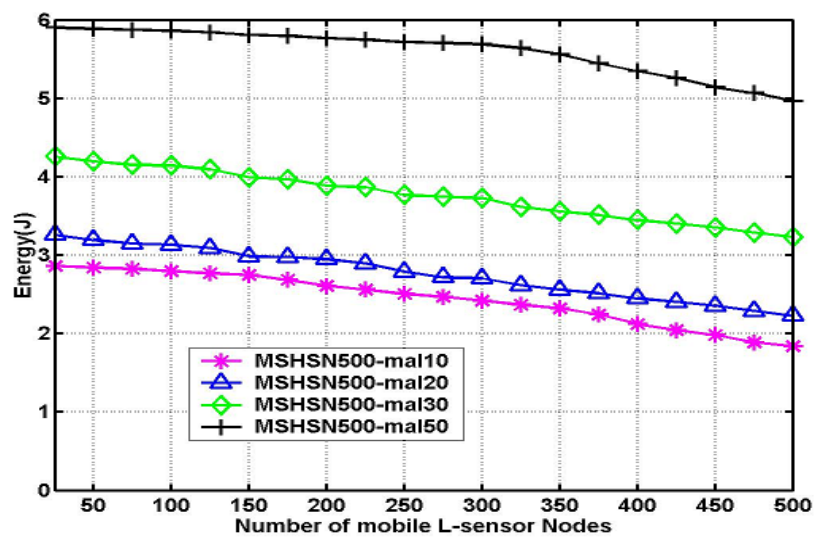

(c).Comparison of Energy Vs number of mobile nodes for various malicious nodes with coverage area $500 \mathrm{~m} \times 500 \mathrm{~m}$

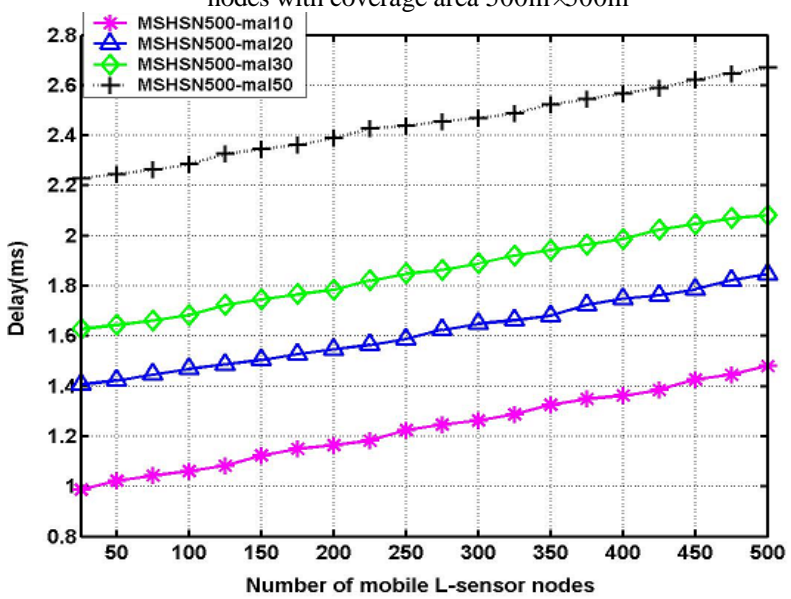

(d).Comparison of Energy Vs number of mobile nodes for various malicious nodes with coverage area $500 \mathrm{~m} \times 500 \mathrm{~m}$

\section{CONCLUSION}

The secured path redundancy algorithm for heterogeneous sensor networks is simulated by varying the number of nodes from 100 to 500 considering fixed and mobile nodes. The two different $(300 \mathrm{~m} \times 300 \mathrm{~m}, 500 \mathrm{~m} \times 500 \mathrm{~m})$ coverage area scenarios are also considered in the simulation for various numbers of malicious nodes ( 30 and 50 ) with mobile sinks (1 to 6). The simulation results prove that secured path redundancy algorithm with sink mobility in heterogeneous sensor networks has better network performance than that of heterogeneous sensor networks of fixed and mobile nodes . The reduction in the energy consumption is achieved by using this algorithm compared to that of heterogeneous sensor networks. The results also demonstrate that the delivery ratio and end to end delay of secured heterogeneous 
sensor network for mobile nodes having mobile sinks is better than the heterogeneous sensor network. The improved performance of this algorithm is due to the usage of a secured alternate path which involves less number of broken paths, hop count and less packet loss to reach the destination node.

\section{REFERENCES}

[1] K. Xu, X. Hong, M. Gerla, "Improving Routing in Sensor Networks with Heterogeneous Sensor Nodes", Proceedings of IEEE Vehicular Technology, Stockholm, Sweden, vol.4, May 2005.

[2] Adrian Perrig, Robert Szewczyk, Victor Wen, David Culler and J.D.Tygar, "SPINS: Security Protocols for Sensor Networks", Proceedings of ACM Annual International Conference on Mobile Computing and Networking, Rome, Italy, July 2001.

[3] Dr.Sami, S. Al-Wakeel and A.Al-Swailem, "PRSA: A Path Redundancy Based Security Algorithm for Wireless Sensor Networks", Proceedings of Wireless Communication and Networking Conference (WCNC 2007), Hong Kong, March 2007.

[4] Vivek Mhatre, Catherine Rosenberg, "Homogeneous vs Heterogenous Clustered Sensor networks: A Comparative Study", Proceedings of IEEE International Conference on Communications (ICC'04), Paris, France, vol.6, pp. 3646-3651, June 2004.

[5] Xiaojiang Du, Sghaier Guizani, Yang Xiao and Hsiao-Hwa Chen, "A Secure Routing Protocol for Heterogeneous Sensor Networks", Proceedings of IEEE GLOBECOM 2006, San Francisco, California, December 2006.

[6] Jeremy Brown and Xiaojiang Du, "Detection of Selective Forwarding attacks in Heterogeneous Sensor Networks", Proceedings of IEEE International Conference on Communications (ICC 2008), Beijing, China, May, 2008.

[7] Xiaojiang Du, Mohsen Guizani, Yang Xiao and Hsiao-Hwa Chen, "Two Tier Secure Routing Protocol for Heterogeneous Sensor Networks", IEEE Transactions on Wireless Communications, Vol. 6,No.9,September 2007.

[8] Chris Karlof and David Wagner, "Secure Routing in Wireless Sensor Networks: Attacks and Countermeasures", Elsevier's Adhoc Networks Journal, Special Issue on Sensor Network Applications and Protocols, Vol-1, pp.293-315, September 2003.

[9] Xiaojiang Du, "Detection of Compromised Sensor Nodes in Heterogeneous Sensor Networks", Proceedings of IEEE International Conference on Communications (ICC'08), Beijing, China, May 2008.

[10] Le Xuan Hung, Ngo Trong Canh, Sungyoung Lee, Young-Koo Lee and Heejo Lee "An Energy-Efficient Secure Routing and Key Management Scheme for Mobile Sinks in Wireless Sensor Networks Using Deployment Knowledge" Journal on Sensors, Vol.8, pp. 7753-7782, December 2008

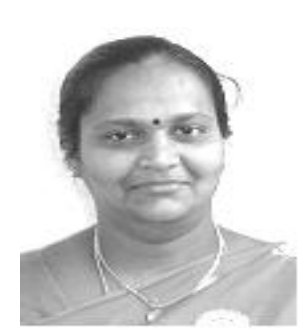

P. Samundiswary received the B.Tech degree (1997) and M.Tech degree (2003) in Electronics and Communication Engineering from Pondicherry Engineering College, India. She is pursuing her Ph.D. programme in the Dept. of Electronics and Communication Engineering, Pondicherry Engineering College affiliated to Pondicherry University, India. She is currently working as Assistant Professor in the Dept. of Electronics and Communication Engineering at Sri Manakula Vinayagar Engineering College affiliated to Pondicherry University, India. Her research interests include wireless communication and wireless sensor networks.

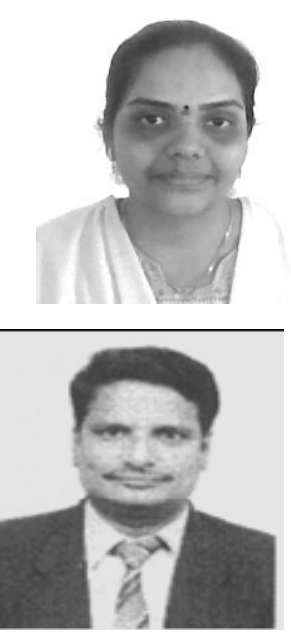

Padma priyadharsini received her B.Tech degree in Electronics and Communication Engineering from Bharathiyar College of Engineering and Technology in 2007. She is pursuing her M.Tech degree in Pondicherry Engineering College, Pondicherry University, India.

P.Dananjayan received B.Sc. from University of Madras in 1979, B.Tech (1982) and M.E. (1984) from the Madras Institute of Technology, Chennai and Ph.D. degree from Anna University, Chennai in 1998. He is working as a Professor in the Dept. of
ECE, Pondicherry Engineering College, India. He is also a visiting professor to AIT, Bangkok. He has more than 60 publications in National and International Journals. He has presented more than 130 papers in National and International conferences. He has produced $7 \mathrm{Ph} . \mathrm{D}$. candidates and is currently guiding eight Ph.D. students. His areas of interest include Spread Spectrum Techniques and Wireless Communication. 